\title{
TI.76.1
}

\section{Topics in Advanced Network Management: Results from a workshop of R\&E network architects}

- Text: internet2-salsa-topics-advanced-network-management-200511.txt

More Information

\begin{tabular}{|l|l|}
\hline Repository ID & Tl.76.1 \\
\hline Persistent URL & http://doi.org/10.26869/TI.76.1 \\
\hline Title & Topics in Advanced Network Management: Results from a workshop of R\&E network architects \\
\hline Authors & Nate Klingenstein, Kevin Miller, Terry Gray, Ken Klingenstein, et al. \\
\hline Sponsor & SALSA \\
\hline Review & \\
\hline Status & Legacy \\
\hline Publish Date & November, 2005 \\
\hline DOI & $10.26869 / T I .76 .1$ \\
\hline Signature & \\
\hline Deprecated & No \\
\hline Future Review & \\
\hline Supersedes & \\
\hline Format & Text \\
\hline Related Docs & \\
\hline Development Location & \\
\hline IP Framework & \\
\hline Subject Tags & middlewarerescue \\
\hline Notes & \\
\hline
\end{tabular}

